\title{
Bacterial Cells Carrying Synthetic Dual-Function Operon Survived Starvation
}

\author{
Yuki Matsumoto, ${ }^{1}$ Yoichiro Ito, ${ }^{1}$ Saburo Tsuru, ${ }^{1}$ Bei-Wen Ying, ${ }^{1}$ and Tetsuya Yomo ${ }^{1,2,3}$ \\ ${ }^{1}$ Department of Bioinformatics Engineering, Graduate School of Information Science and Technology, Osaka University, \\ 1-5 Yamadaoka, Suita, Osaka 565-0871, Japan \\ ${ }^{2}$ Graduate School of Frontier Biosciences, Osaka University, 1-5 Yamadaoka, Suita, Osaka 565-0871, Japan \\ ${ }^{3}$ Exploratory Research for Advanced Technology (ERATO), Japan Science and Technology Agency (JST), Saitama 332-0012, Japan
}

Correspondence should be addressed to Tetsuya Yomo, yomo@ist.osaka-u.ac.jp

Received 30 May 2011; Accepted 1 September 2011

Academic Editor: George E. Plopper

Copyright () 2011 Yuki Matsumoto et al. This is an open access article distributed under the Creative Commons Attribution License, which permits unrestricted use, distribution, and reproduction in any medium, provided the original work is properly cited.

A synthetic dual-function operon with a bistable structure was designed and successfully integrated into the bacterial genome. Bistability was generated by the mutual inhibitory structure comprised of the promoters $\mathrm{P}_{\text {tet }}$ and $\mathrm{P}_{\text {lac }}$ and the repressors LacI and TetR. Dual function essential for cell growth was introduced by replacing the genes (i.e., hisC and leuB) encoding proteins involved in the biosynthesis of histidine and leucine from their native chromosomal locations to the synthetic operon. Both colony formation and population dynamics of the cells carrying this operon showed that the cells survived starvation and the newly formed population transited between the two stable states, representing the induced his $C$ and leuB levels, in accordance with the nutritional status. The results strongly suggested that the synthetic design of proto-operons sensitive to external perturbations is practical and functional in native cells.

\section{Introduction}

Synthetic approaches are widely employed to observe novel phenomena in living organisms $[1,2]$, particularly in studies on species sustenance and population adaptation [3-6]. As a fundamental characteristic of living systems, adaptation is generally achieved by well-known sensing mechanisms, such as operons and regulons, which have been described in detail [7-9]. These mature systems and/or pathways for survival have arisen by evolution [10]. Genetic disturbance generally subjects cells to vital stress and often trigger population extinction. However, recent pioneering studies on genetic rewiring showed that cells have great potential to survive under severe conditions regardless of the disruption of native regulation $[5,6,11]$. Therefore, in addition to the known evolved sensing mechanisms, stochastic survival strategies have recently been proposed as a complementary route for cells to achieve adaptation [12-14]. In these studies, genetic reconstruction was performed to avoid interruption by the native genetic structures and to force living organisms to use foreign or premature genetic modules to achieve adaptation.

Due to the difficulties in genetic construction [15], there are few examples of synthetic operons contributing to cellular functions that are physiologically essential for living cells. The studies were generally based on the plasmid format of multiple copies, which was quite different from the genome format of only a single copy [16]. The copy number of the genetic material contributes markedly to phenotypic variations in a genetically identical population [17-19]. There has been a great deal of recent progress in techniques for constructing stable gene circuits $[20,21]$. Efficient and precise genome recombination for complex synthetic circuits has been reported previously [16]. This method allows functional synthetic operons to be built in living bacterial cells and to be replicated along with the genome, leading to a stable genetically heritable state. Studies on the synthetic operons used by native cells responsive to external changes were reliable, due to the reduced fluctuation 


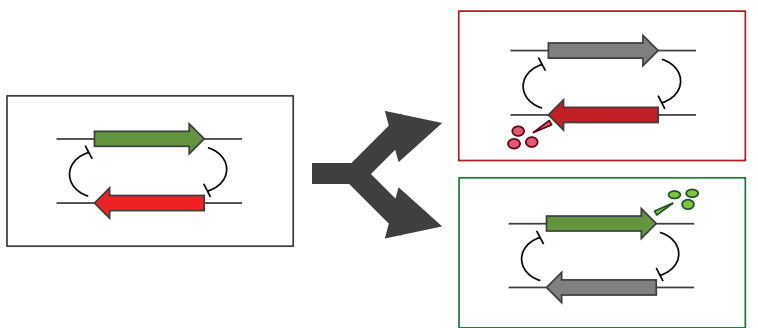

(a)



(b)

FIGURE 1: Genetic design and construction of the synthetic dual operon. (a) Design principle. The mutually inhibitory circuit generally provides two stable states (red and green frames) for the cells. Only one fragment in the dual operon (red and green arrows) can be activated and achieves one of the two essential biological roles (red and green circles). (b) Genetic construction. The synthetic dual operon (in red and green) presenting two physiological roles (leucine and histidine biosynthesis, resp.) essential for cell growth was integrated into the $E$. coli genome. leuB and hisC expression were controlled by lacI and tetR, and reported by $m$ Cherry (red fluorescent protein) and gfpuv5 (green fluorescent protein), respectively. The genes lacI-Y, leuB and his C within their native operons (Lac operon, Leu operon and His operon) were genetically disrupted.

in genetic copy number and the increased stability of the synthetic design [16].

The induced and suppressed expression of synthetic operons can be designed according to the physical principles described in detail previously $[22,23]$. Monostable $[24,25]$ and multistable $[3,12,13]$ structures of genetic circuits are available for genetic design. In general, the bistable structure, providing two stable states for the cells, has a fixation effect on the stable state, and it is therefore feasible for experimental observation and is widely used $[3,12$, 13]. Positive feedback and mutual inhibitory features can be employed as design principles for constructing dualfunction operon-like genetic circuits to achieve two distinct phenotypes. This genetic structure allows the cells to transit between two stable states stochastically, and permits analysis of whether and how the synthetic operon functions in living cells and its role in adaptation.

In the present study, two phenotypes of two diverse biological functions were designed, both of which result in a fitness recovery depending on the external conditions. Here, the functions of leucine and histidine biosynthesis were introduced into the synthetic operon. In general, Escherichia coli cells use the Leu operon and His operon to respond to starvation [26-28]. Depletion of leucine will lead to the induced expression of structural genes in the Leu operon; similarly, histidine depletion will cause an increase in expression of proteins encoded within the His operon $[8,26]$. The genes, leuB and his $C$, which are located within the Leu and His operons [7], are responsible for leucine and histidine biosynthesis, respectively. Rewiring these stringent starvation genes to the synthetic operon not only disturbs the original native regulation but also introduces physiological activities for the synthetic operon. Here, we investigated whether the synthetic operon comprised of the rewired genes could respond to a transition in nutritional status. The results showed that bacterial cells carrying this synthetic operon formed diverse populations in response to the nutritional conditions and survived under conditions of nutrient depletion. This is the first demonstration of a genome-integrated dual-function synthetic operon sensitive to an environmental transition. It strongly suggested that the synthetic design of proto-operons sensitive to external perturbations is practical for native cells.

\section{Results and Discussion}

2.1. Design Principle and Genetic Construction of the Synthetic Operon. Bistability was introduced into the genetic design of the synthetic operon to produce two discrete stable states (Figure 1(a), illustrated in red and green), which were prepared for two different biological functions essential for cell growth. A positive feedback structure would accelerate the expression of any of the two expression units (Figure 1(a), red and green arrows) occasionally showing slightly higher expression level, while the mutual inhibitory structure would suppress expression of the other unit, leading to a fixation effect of the expressed unit $[3,22,29]$. Once genes involved in physiological functions responsive to external changes are introduced into the two expression units (Figure 1(a), red and green cycles), the selective expression of the two units in cells can be considered a synthetic operon contributing to survival and/or adaptation (Figure 1(a), red and green frames). The synthetic operon that was finally designed is shown in Figure 1(b) and was integrated into the E. coli genome at a constant copy number.

The two expression units, indicated in red and green, representing a dual-function synthetic operon, were built in the E. coli genome at the intC and galK sites. The red expression unit consisted of three genes, mCherry, tet $R$, and $l e u B$, encoding a red fluorescent protein (RFP), a repressor protein for blocking expression of the green unit (the promoter $\mathrm{P}_{\text {tet }}$ ), and an enzyme contributing to leucine biosynthesis, respectively. The green expression unit contained three genes, gfpuv5, lacI, and hisC, encoding a green fluorescent protein (GFP), a repressor protein 
inhibiting expression of the red unit (the promoter $\mathrm{P}_{\text {lac }}$ ), and an enzyme involved in histidine biosynthesis, respectively. To oblige the cells to use the functional genes, his $C$ and leuB, within the synthetic operon, the native regulation of Leu and $H i s$ operons was disturbed by removing leuB and his $C$ from their native chromosomal locations. Thus, the expression of $l e u B$ and his $C$ only inside the synthetic operon was reported by the red and green fluorescence, respectively. In addition, to prevent disturbance of synthetic operon expression (from the promoter $\mathrm{P}_{\mathrm{lac}}$ ) by the endogenous level of LacI protein, the native lacI gene and the related genes lacY and lac $Z$ were deleted from the genome, as shown in Figure 1(b). A synthetic operon based on a mutual inhibitory circuit and showing two discrete physiological states, leucine (red) and histidine biosynthesis (green), was constructed on the E. coli chromosome (Figure 1(b)) by homologous recombination as described previously [16].

According to the design principle, the E. coli cells carrying this synthetic operon could show two phenotypes, red and green, representing high-level expression of leuB and hisC, respectively. These two phenotypes were related to two physiological functions, that is, leucine and histidine biosynthesis, respectively. Bistability, resulting from the mutual inhibitory properties of the synthetic operon, was assumed to confer the so-called "memory effect" on the cells carrying this structure, according to a previous study using the same promoter and repressor cassette [16]. That is, the cells were thought to be able to show two distinct phenotypes under identical culture conditions due to the diverse histories (induction) of gene expression. However, as the cells had additional deletion of multiple genes and gene rewiring, it was necessary to confirm that the synthetic operon did actually work according to the design principle. In addition, whether the rewired genes contributed to amino acid biosynthesis and cell survival under conditions of starvation was unknown.

2.2. Dual Function of the Synthetic Operon in E. coli Cells. The engineered cells grown in minimal medium M63 containing $1 \mathrm{mM}$ histidine and $1 \mathrm{mM}$ leucine showed equivalent growth rate regardless of the induction conditions (Figure 2(a)), suggesting that the large reconstruction at the chromosomal level did not disturb their growth. Microscopic observations showed that the cells displayed green fluorescence when growing without any inducers (Figure 2(c), top), indicating that the synthetic operon showed preferential high-level expression of the green unit controlled by $\mathrm{P}_{\text {tet }}$. The cells showed either red or green fluorescence on induction by isopropyl $\beta$-D-1-thiogalactopyranoside (IPTG) or doxycycline (Dox), respectively (Figure 2(c), middle and bottom). Cell populations formed under varied inducing conditions showed a distribution of higher fluorescence intensity of either red or green (Figure 2(b)), consistent with microscopic observations (Figure 2(c)). The results verified that the synthetic operon allowed the cells to show either the red or green phenotype according to the inducing conditions in amino acid-supplemented medium (Figures 2(b)-2(c)). No intermediate phenotype (e.g., yellow) was detected by varying the concentrations of the inducers. These observations indicated that the binding affinities between promoters and repressors were strong enough to avoid leakage of gene expression, and the synthetic operon provided two stable states for the cells. Thus, the functional mutual inhibitory structure (promoters and repressors) was clearly confirmed.

Next, we examined whether the specific induction of a single unit, red or green, would rescue the cells from starvation. The cell cultures were all initiated from a population of $\sim 10^{3}$ cells $/ \mathrm{mL}$. The full induction of $\mathrm{P}_{\text {lac }}(100 \mu \mathrm{M}$ IPTG $)$ triggered the cells to turn red (Figures 2(b)-2(c)), that is, induced expression of leuB. The cells supplied histidine in the growth medium succeeded in population propagation and showed a relatively high growth rate (Figure $2(\mathrm{~d})$ ). In contrast, cells cultured in leucine-containing medium failed to grow $\left(<0.01 \mathrm{~h}^{-1}\right)$, which was likely due to the depletion of histidine. As the expression of tet $R$ was highly induced, the gene essential for histidine biosynthesis (hisC) was strongly suppressed, leading to severe starvation from histidine depletion. Similarly, the addition of $100 \mathrm{nM}$ Dox resulted in high-level expression of $\mathrm{P}_{\text {tet }}$ and caused the cells to form a population showing green fluorescence (Figure 2(b)$2(c))$. As a consequence, the highly induced expression of his $C$ allowed growth only under conditions in which leucine was supplied (Figure 2(d)). The results demonstrated that the induced expression of either single unit in the synthetic operon could compensate for the depletion of the corresponding amino acid. These observations indicated that the dual function of the synthetic operon worked successfully in living cells. Note that the higher concentrations of inducers (either IPTG or Dox) led to inhibition of cell growth. The depletion of both amino acids caused vital stress for the cells and led to a pause in growth.

\subsection{Bistable Conditions for Selective Expression of the Synthetic} Operon. As $30 \mu \mathrm{M}$ IPTG was sufficient to fully induce the red unit, as confirmed by flow cytometry (data not shown), hysteresis of the synthetic operon was investigated under these induced conditions. Cells preliminarily grown in the absence or presence of IPTG $(30 \mu \mathrm{M})$ were transferred to fresh medium containing various concentrations of IPTG from 0 to $30 \mu \mathrm{M}$, with an initial cell concentration of $\sim 10^{3}$ cells $/ \mathrm{mL}$. As shown in Figure 3(a), the newly formed cell populations showed distinct expression patterns, even under identical induction conditions (i.e., $0-15 \mu \mathrm{M}$ IPTG), which was due to the differences in preliminary culture conditions. The trajectories of averaged fluorescence intensity kept different even without induction (Figure 3(b)), suggesting that the hysteresis was quite strong. Serial transfer experiments showed that the memory effect could last for 3 days (equivalent to $\sim 45$ generations, data not shown). Hysteresis was confirmed not only by population analysis using flow cytometry but also by colony formation assay on agar plates. Approximately 100 cells with the same preculture conditions (in the absence or presence of IPTG) were inoculated onto agar plates containing various concentrations of IPTG $(0-$ $30 \mu \mathrm{M})$. Formation of single colonies was observed after 2 days of incubation. The new colonies with a history of red induction maintained the red phenotype even with an IPTG concentration as low as $10 \mu \mathrm{M}$ (Figure 3(c)), in agreement 


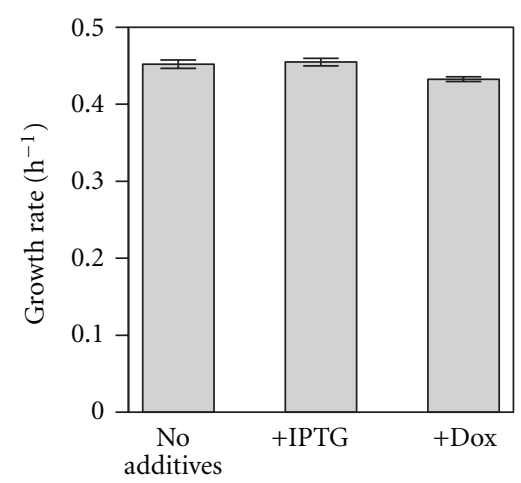

(a)

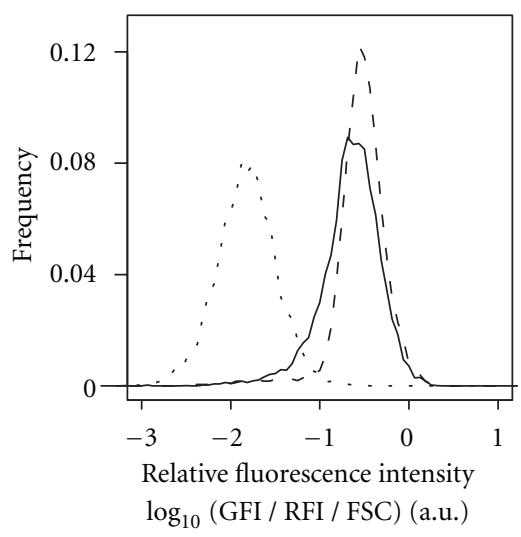

(b)


(c)

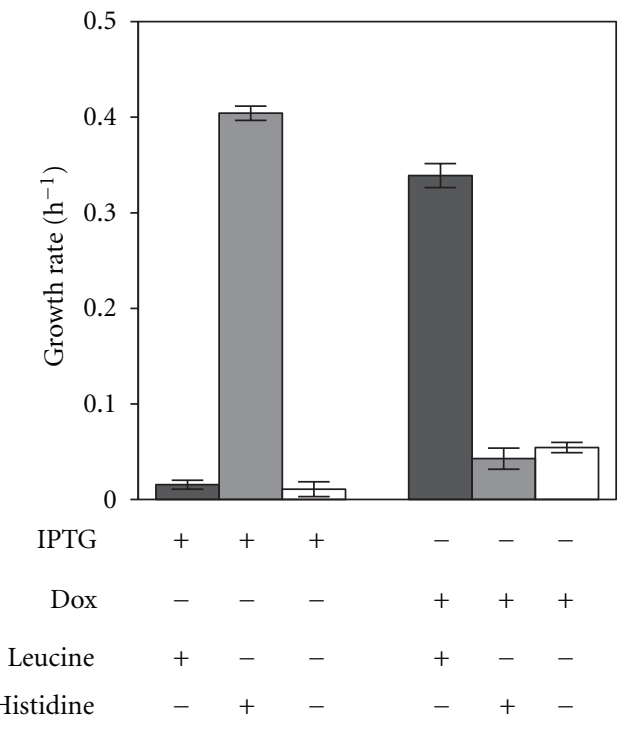

(d)

FIgURE 2: Regulation and function of the synthetic dual operon. (a) Cell growth. E. coli cells carrying the synthetic operon grown under the amino acid-supplied conditions showed a constant growth rate regardless of the induction conditions. No additives, + IPTG and + Dox represent the absence of inducers, the presence of $100 \mu \mathrm{M}$ IPTG and the presence of $100 \mathrm{nM}$ doxycycline, respectively. (b) Gene expression. Population distributions of relative fluorescence intensity in the absence of inducers (solid line), in the presence of $100 \mu \mathrm{M}$ IPTG (dotted line), or in the presence of $100 \mathrm{nM}$ Dox (dashed line). Green fluorescence intensity (GFI), red fluorescence intensity (RFI) and forward scattering (FSC) represent the abundances of GFP and RFP expressed in single cells and the relative cell size, respectively. GFI/RFI/FSC indicates the concentration of GFP bias in cells. (c) Microscopic observation. Fluorescence images of cells grown in the absence of inducers (top), in the presence of $100 \mu \mathrm{M}$ IPTG (middle) or in the presence of $100 \mathrm{nM}$ Dox (bottom) are shown. The scale bars represent $10 \mu \mathrm{m}$. (d) Physiological function. E. coli cells carrying the dual operon were cultured under various nutritional and induced conditions. Cell growth was examined after $24 \mathrm{~h}$ in culture under each condition. + and - indicate the presence and absence of the additives, respectively. The concentrations of IPTG, Dox, leucine, and histidine were $100 \mu \mathrm{M}, 100 \mathrm{nM}, 1 \mathrm{mM}$, and $1 \mathrm{mM}$, respectively.

with the results of population analysis (Figure $3(a))$. The ratio of green colonies among all newly formed colonies was different between the initial two populations under conditions of $5-15 \mu \mathrm{M}$ IPTG (Figure 3(d)). These observations indicated that the cells could form either red or green populations under bistable conditions (i.e., 5-15 $\mu \mathrm{M}$ IPTG). The differences in the bistable ranges at the colony and population levels were due to the diverse time scales used, that is, 1 night for population dynamics analysis and 2 days for colony formation assay. As the two stable states linked to two independent physiological functions, the stochastically selective expression (red or green) of the synthetic operon could potentially help the cells to survive under conditions of histidine or leucine depletion.

2.4. Colony Formation in Response to Environmental Transition. The cells carrying the synthetic operon were subjected to the environmental transition to examine whether and what type of colonies could be formed on agar plates with varied nutritional conditions. Cells preliminarily grown in amino acid-containing media were transferred onto agar plates containing $15 \mu \mathrm{M}$ IPTG (bistable conditions) but various concentrations of amino acids. Aliquots of $\sim 100$ cells were inoculated onto three different plates containing both amino acids, only leucine, or only histidine. Colony formation was observed under the microscope and followed for 1 week. The cells with hisC-induced memory (green) formed all green colonies on plates containing both histidine and leucine and on those containing only leucine (+His + Leu and -His + Leu, resp.; Figure 4(a)), in comparison to the red colonies formed on leucine-depleted plates (+His - Leu; Figure 4(a)). The red colonies were born from the initial green colony, indicating a transition between the two expression units in the synthetic operon 


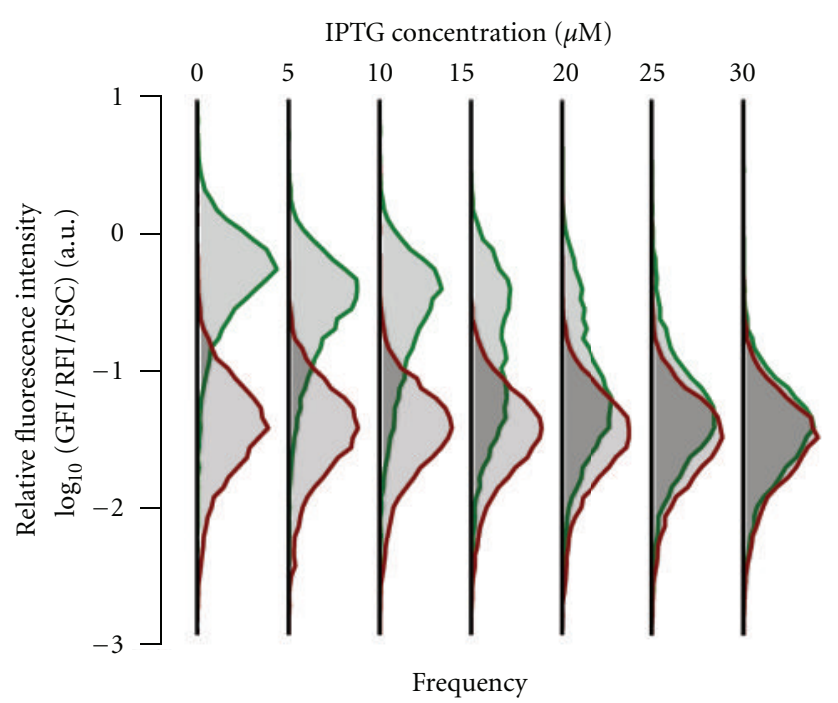

(a)



(c)



(b)

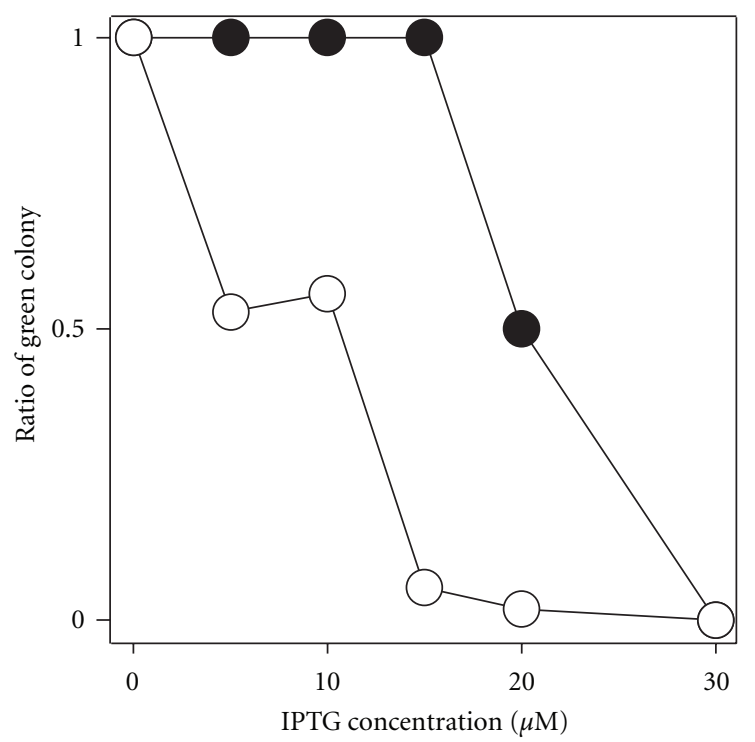

(d)

FIgURE 3: Hysteresis observed at population and colony levels. (a) Population distributions of relative fluorescence intensity. The cells initially showing only green (IPTG $0 \mu \mathrm{M}$ ) or red (IPTG $30 \mu \mathrm{M}$ ) fluorescence (illustrated as green or red distributions, resp.) were cultured under amino acid-supplied conditions and in the presence of various concentrations of IPTG (from 0 to $30 \mu \mathrm{M}$ in 5 - $\mu \mathrm{M}$ increments). Distributions of the newly formed populations are shown in green and red in accordance with the initial expression status. (b) Trajectories of averaged expression level. The relative concentrations of GFP bias (GFI/RFI/FSC) were calculated from the distributions shown in (a). The cells precultured in IPTG-free (closed circles) or IPTG-supplied (30 $\mu \mathrm{M}$ IPTG, open circles) medium showed different trajectories of averaged concentrations of GFP bias. (c) Fluorescence images of colony formation. The cells initially grown in IPTG-free or IPTG-supplied (30 $\mu \mathrm{M}$ ) medium were inoculated onto the agar plates containing various concentrations of IPTG (from 0 to $30 \mu \mathrm{M}$ ). Aliquots of approximately 100 cells were inoculated on each plate. The newly formed single colonies were observed under a microscope. NIBA and WIG indicate the filters used to detect green and red fluorescence, respectively. (d) Ratio of green and red colonies. The cells initially grown in IPTG-free (closed circles) or IPTG-supplied (30 $\mu \mathrm{M}$ IPTG, open circles) medium showed different ratios of the newly formed green and red colonies.

(see Figure S1 in supplementary materials available online at doi:10.1155/2011/489265). Delay in colony formation due to leucine depletion was observed, suggesting that the stochastic appearance of fit cells with the adaptive expression pattern was rare. Similarly, the initial leuB-induced cells formed red colonies on plates containing both histidine and leucine and on those containing only histidine $(+\mathrm{His}+\mathrm{Leu}$ and +His - Leu, resp.; Figure 4(b)), in comparison to the red colonies formed on histidine-depleted plates ( $-\mathrm{His}+\mathrm{Leu}$; Figure $4(\mathrm{~b}))$. In conclusion, the cells carrying the synthetic 


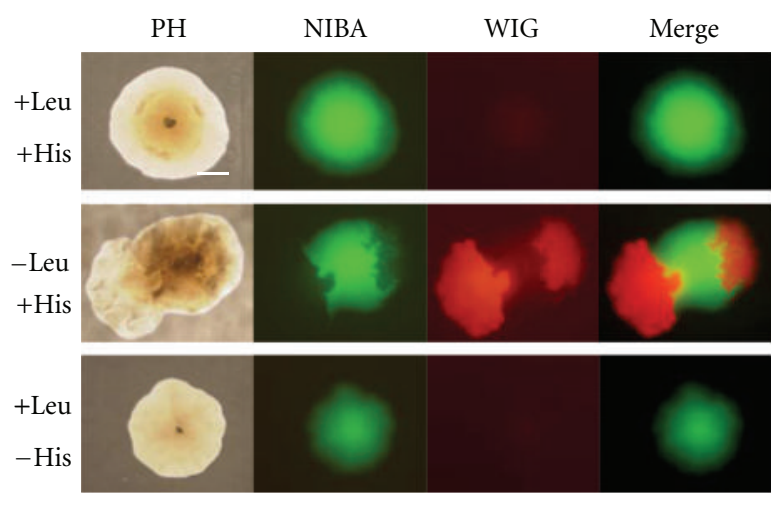

(a)

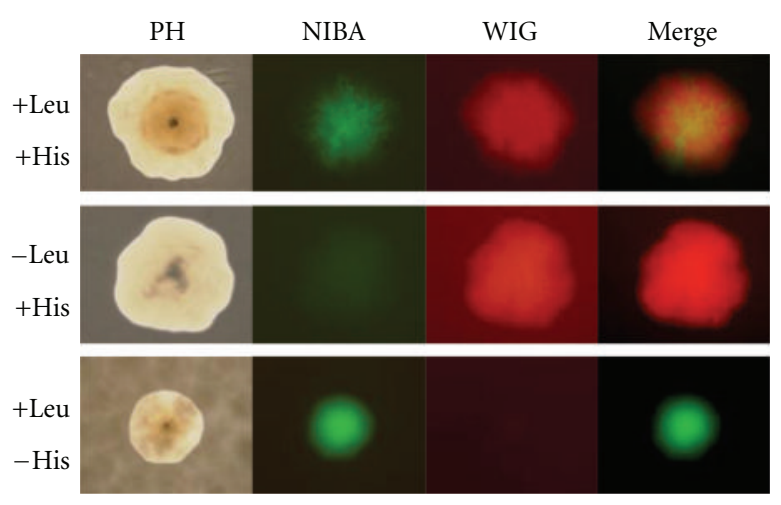

(b)

FIGURE 4: Colony formation under varied nutritional conditions. E. coli cells carrying the synthetic dual operon preliminarily grown under IPTG-free (a) and IPTG-supplied ( $30 \mu \mathrm{M}$ IPTG, (b)) conditions were inoculated onto M63 agar plates containing $15 \mu \mathrm{M}$ IPTG with different amino acid contents. The newly formed single colonies were observed by fluorescence microscopy after 3-5 days of incubation. NIBA and WIG are described in Figure 3. PH and Merge indicate the phase-contrast and merged images of NIBA and WIG, respectively. The final concentrations of amino acids were all $1 \mathrm{mM}$. + and - indicate the presence and absence of leucine (Leu) and histidine (His), respectively.

operon formed colonies of two discrete phenotypes, induced leuB (red) or hisC (green), in response to the nutritional conditions.

2.5. Growth Recovery along with Selective Expression of the Synthetic Operon. We analysed population dynamics to evaluate the relation between cell growth and expression of the synthetic operon. The cells preliminarily grown in the presence of both amino acids were washed and transferred into fresh media containing $5 \mu \mathrm{M}$ IPTG for induction (the bistable condition; Figures 3(a)-3(b)), but differing in the amounts of both amino acids ( $1 \mathrm{mM}$ of each). The initial cell concentration was $\sim 10^{6}$ cells $/ \mathrm{mL}$, which was both low enough to avoid cross feeding and high enough for precise measurement of subpopulations within a single population. Time sampling of the cell culture was performed at $2-\mathrm{h}$ intervals $(0-14 \mathrm{~h}$; extended observation till 22 or $30 \mathrm{~h}$ was performed under slow growth conditions). The increases in cell concentrations (growth curves) showed that the cell population with the memory of induced his $C$ expression (green unit) grew in media to which only leucine was added as fast as in that containing both amino acids, but showed a long time lag in growth recovery when grown in the absence of leucine (Figure 5(a), upper). The distributions of the growing populations in relative expression level remained at the initial high hisC (green) levels in the presence of leucine regardless of the presence or absence of histidine (Figure 5(b), upper, left, and middle). In contrast, the cell population gradually shifted from the initial high hisC (green) level to the high leuB (red) level in leucine-depleted medium (Figure 5(b), upper, right).

On the other hand, the cell population with the memory of induced leuB expression (red unit) showed relatively high growth rates in media containing histidine, but slower growth in the absence of histidine (Figure 5(a), bottom). The distributions of relative expression remained in the initial high leuB (red) level in the presence of histidine but shifted to the high hisC (green) level under histidine-depleted conditions (Figure 5(b), bottom). No such population transition was detected in the control strain (Figure S1A), which had the same genetic structure but without the gene rewiring, as described previously [16]. The cell population either with the initial high RFP level or with the initial high GFP level retained the steady distribution identical to that seen initially along with the population propagation (Figure S1B). That is, the cell population carrying the synthetic operon changed its expression state in response to starvation, in comparison to the control strain in which the population remained in the initial state regardless of the nutritional status. These results indicated that the synthetic operon sensitive to changes in the external environment played a role in population adaptation.

Furthermore, the relation between growth rate and averaged expression level of the population was evaluated. The cell growth rates were evaluated according to the cell concentrations between every 2 sampling points. The growth rate showed only small fluctuations and the relative expression level remained approximately constant when cells with hisC-induced memory were grown in the presence of leucine (Figure 5(c), upper, left, and middle). In contrast, the growth rate dropped off markedly under conditions of leucine depletion but recovered gradually, accompanied by an increase in leuB (red) expression level (Figure 5(c), upper, right). In addition, the temporal trajectory of the relation between cell growth and relative expression level in the cell population with leuB-induced memory indicated a significant reduction in growth rate with histidine depletion, but rapid recovery along with the gradual increase in hisC (green) expression level (Figure 5(c), bottom). These observations provided clear experimental evidence that selective expression of the synthetic operon contributed to population adaptation by transition between the two stable states of biological functions.

The growth recovery and population transition due to histidine depletion were faster than those due to leucine 




(a)
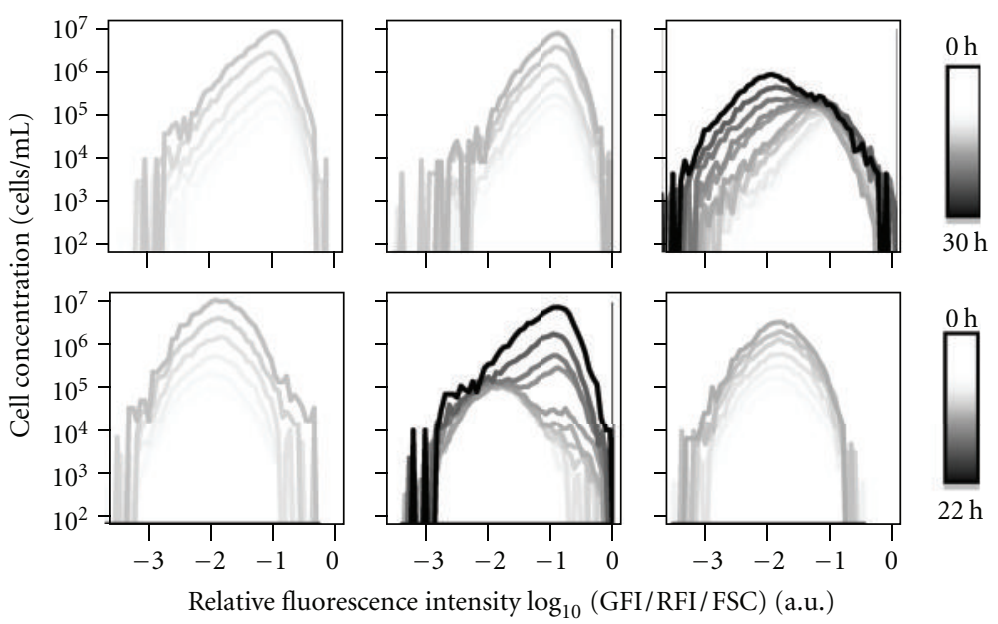

(b)
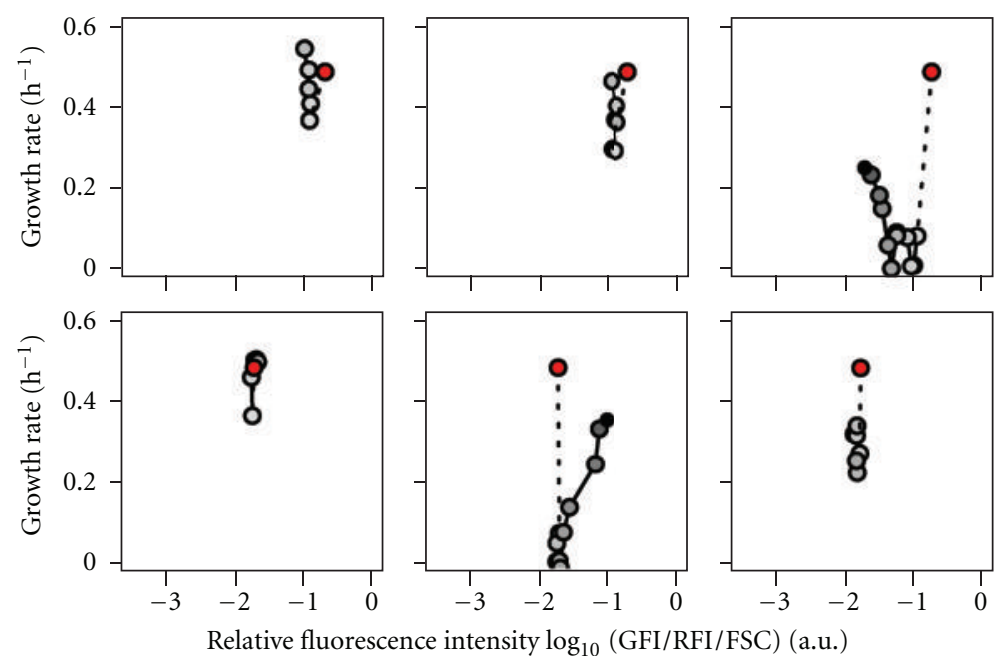

(c)

FIGURE 5: Temporal changes in population distribution and cell growth. (a) Cell growth. Temporal changes in cell concentration were examined at 2-h intervals (0-14 h; extension till 22 or $30 \mathrm{~h}$ in slow growth conditions). Closed and opened symbols represent the cell populations born from the green and red initial states, respectively. Circles, squares, and triangles indicate growth in the presence of both amino acids and with single addition of leucine or histidine, respectively. (b) Temporal changes in the distribution of relative fluorescence intensity. Cells preliminarily grown in the presence or absence of IPTG were approximately inoculated $10^{6}$ cells/mL into fresh media containing $5 \mu \mathrm{M}$ IPTG under different nutritional conditions. Timed sampling of the cell culture was performed at 2-h intervals (except from 14 to $22 \mathrm{~h}$ ). Cell concentration and expression level were measured by flow cytometry. The distributions of newly formed populations (from 0 to $30 \mathrm{~h}$ or $22 \mathrm{~h}$ ) are shown from light to dark grey, respectively. Population propagation with the addition of both amino acids or with the single addition of $1 \mathrm{mM}$ leucine or $1 \mathrm{mM}$ histidine are shown in the left, middle and right panels, respectively. (c) Relationship between growth and expression. The growth recovery trajectory is indicated with respect to the temporal changes in distribution shown in (a) and (b). Circles filled in red indicate the $0 \mathrm{~h}$ time point. 
depletion. We assumed that the green unit (hisC) was much easier to induce than the red unit $(\operatorname{leu} B)$, which was consistent to the inherent properties of the synthetic operon, that is, preferential expression of the green unit in rich medium without any inducers (Figures 2(b)-2(c)). There are several possible reasons for these observations, such as slight leakage of gene expression from $\mathrm{P}_{\text {tet }}$, essential amino acid requirement, as yet unknown synchronised expression changes in other related genes, and so forth. Further studies of other genes and/or amino acids are required to determine the universality of the capacity of the synthetic operon to respond to external perturbations.

\section{Materials and Methods}

3.1. Strains and Plasmids. The E. coli strain DH1 was used for synthetic construction. The genes lacI, lacY, lacZ, leuB and his $C$ were disrupted by homologous recombination as described previously $[16,30]$. The fragment carrying $r f p$ (mCherry, Clontech), tetR, leuB, and cat genes under the control of the lac promoter $\left(\mathrm{P}_{\mathrm{lac}}\right)$ was first constructed in the plasmid pBRintCleuB2, and finally inserted into the int $C$ site on the genome. Similarly, the fragment containing gfp ( $g f p u v 5)$, lacI, hisC and kan genes under the control of the tet promoter $\left(\mathrm{P}_{\text {tet }}\right)$ was constructed in the plasmid pBRgalKhisC3, and was subsequently inserted into the galK site on the genome. The DNA fragments in the plasmids were flanked by a genome sequence of 300-500 bp with neighbouring intC or galK genes as reported previously [16]. The plasmids pBRintCleuB2 and pBRgalKhisC3 were constructed by inserting leuB, and his $C$ (amplified from the DH1 genome) into the plasmids pBRintCs and pBRgalKGR (pBR322 derivative) [16] at the HindIII site, respectively. Homologous sequences were generated by polymerase chain reaction (PCR) elongation, and the primers used to amplify the target DNA fragments for genome replacement at the chromosomal locations of int $C$, and galK were described previously [16].

3.2. PCR, Enzymes, and DNA Purification. The DNA fragments used for genome replacement were amplified using Phusion (New England BioLabs, Neb), and colony PCR confirmation was carried out using GoTaq (Promega) according to the respective manufacturer's protocols. Restriction enzymes and biochemical reagents used for genetic construction were obtained commercially from Takara Bio and NEB. DNA purification was performed using commercial kits, spin columns or gel extraction (QIAquick and MinElute; Qiagen).

3.3. Cell Culture. Bacterial cells were grown in minimal medium (M63: $62 \mathrm{mM} \mathrm{K}_{2} \mathrm{HPO}_{4}, 39 \mathrm{mM} \mathrm{KH} \mathrm{KO}_{4}, 15 \mathrm{mM}$ $\left(\mathrm{NH}_{4}\right)_{2} \mathrm{SO}_{4}, 2 \mu \mathrm{M} \mathrm{FeSO}{ }_{4} \cdot 7 \mathrm{H}_{2} \mathrm{O}, 15 \mu \mathrm{M}$ thiamine hydrochloride, $203 \mu \mathrm{M} \mathrm{MgSO} \cdot 7 \mathrm{H}_{2} \mathrm{O}$, and $22 \mathrm{mM}$ glucose) in the presence of $1 \mathrm{mM}$ leucine and/or $1 \mathrm{mM}$ histidine. Cells were cultured at $37^{\circ} \mathrm{C}$ for several passages until the growth rate became stable. Full expression of RFP and GFP was induced in the presence of $100 \mu \mathrm{M}$ isopropyl $\beta$-D-1-thiogalactopyranoside (IPTG) and $100 \mathrm{nM}$ doxycycline. For confirmation of hysteresis and observation of the response to nutrient depletion, bacterial cells were precultured with 0 or $30 \mu \mathrm{M}$ IPTG to induce the expression of gfpuv5-lacI-hisC or mCherrytetR-leuB, respectively. Precultured cells were inoculated into fresh liquid and/or agar mM63 medium with various concentrations of IPTG $(0,5,10,15,20,25$, and $30 \mu \mathrm{M})$. The initial cell concentration was approximately $10^{3}$ cells $/ \mathrm{mL}$, and the cell cultures were examined by flow cytometry after 24 -h incubation at $37^{\circ} \mathrm{C}$. Single colonies formed on agar medium were observed by fluorescence microscopy after 48 $\mathrm{h}$ incubation at $37^{\circ} \mathrm{C}$. Approximately 100 cells were initially inoculated onto each agar plate. The growth rate $\left(\mathrm{h}^{-1}\right)$ was calculated according to the initial $\left(C_{\text {init }}\right)$ and final $\left(C_{\text {fin }}\right)$ cell concentrations and the culture time $(T)$, according to the following formula: $\ln \left(C_{\text {fin }} / C_{\text {init }}\right) / T$.

3.4. Microscopic Observation. Bacterial cells in the logarithmic growth phase ( $1 \mu \mathrm{L}$ of culture) were placed on glass coverslips and immersed in the same medium as used for preculture. For colony observation, 1.5\% agar plates using M63 medium were directly placed on the stage. Fluorescence images were acquired at $4 \times$ magnification using a fluorescence microscope (IX70; Olympus) and a cooled CCD colour camera (VB7010; Keyence). The filter sets of $470-495 \mathrm{~nm}$ excitation, $510-550 \mathrm{~nm}$ emission and 530-550 nm excitation, $>575 \mathrm{~nm}$ emission were used for measuring fluorescence intensity from $g f p u v 5$ and mCherry, respectively.

3.5. Flow Cytometry. Expression level of GFP and RFP (fluorescence intensity) and relative cell size were evaluated using a flow cytometer (FC500; Beckman Coulter) with a $488 \mathrm{~nm}$ argon laser. Band pass filters of 525/20 and 620/20 nm were used for detection of green and red fluorescence, respectively. The following PMT voltage settings were applied: forward scatter (FSC), 900; side scatter (SSC), 350; GFP, 750, RFP, 670 . In each tube, about 10,000 cells were measured and the flow data were analysed by custom-designed scripts written in R [31]. Systematic errors resulting from events that occurred at the extremes of the range of the instrument were eliminated. Cell samples mixed with fluorescent beads $(2.76 \mu \mathrm{m}$ Fluoresbrite YG Microspheres; Polysciences) were loaded for calculation of cell concentration.

3.6. Nutrient Depletion. Bacterial cells were grown in M63 medium supplemented with $1 \mathrm{mM}$ leucine, $1 \mathrm{mM}$ histidine and various concentrations of IPTG for $24 \mathrm{~h}$ at $37^{\circ} \mathrm{C}$ as described above. Aliquots of $500 \mu \mathrm{L}$ of exponentially growing cells $\left(\sim 10^{8}\right.$ cells $\left./ \mathrm{mL}\right)$ were harvested in spin columns $(0.2 \mu \mathrm{m}$ Ultrafree-MC Centrifugal Filter Units; Millipore) by centrifugation at 5,000 rpm (or $2,300 \times \mathrm{g}$ ) for $60 \mathrm{~s}$. After discarding the flow-through fraction, the cells were washed with $500 \mu \mathrm{L}$ of fresh medium without amino acids. After repeating centrifugation and washing processes, the concentration of the cell suspension was determined by flow cytometry. The initial cell concentration subjected to starvation was approximately $10^{6}$ cells $/ \mathrm{mL}$. 


\section{Conclusions}

A dual-function synthetic operon was designed and integrated into the bacterial chromosome. The experiments demonstrated that the bacterial cells used this synthetic operon in response to nutritional transition and survived starvation. Successful achievement of population adaptation dependent on such external regulation not only indicated that the synthetic design could play an important role in sustenance and adaptation, but also suggested that it could be universally applied by living systems. As such adaptive population transition could be both due to switching of selective expression and caused by the proliferation of adaptive cells, further analyses of the emergence of first born switched cells and estimation of the switching rate are required. Such synthetic approaches will likely yield further novel findings in living systems in future studies.

\section{Acknowledgments}

The authors thank Natsuko Yamawaki, Junko Asada, and Natsue Sakata for technical assistance. This work was partially supported by Grants-in-Aid for Challenging Exploratory Research no. 22657059 (to B-W. Ying) and the "Global $\mathrm{COE}$ (Centers of Excellence) program" of the Ministry of Education, Culture, Sports, Science and Technology, Japan.

\section{References}

[1] R. McDaniel and R. Weiss, "Advances in synthetic biology: on the path from prototypes to applications," Current Opinion in Biotechnology, vol. 16, no. 4, pp. 476-483, 2005.

[2] J. Hasty, D. McMillen, and J. J. Collins, "Engineered gene circuits," Nature, vol. 420, no. 6912, pp. 224-230, 2002.

[3] A. Kashiwagi, I. Urabe, K. Kaneko, and T. Yomo, "Adaptive response of a gene network to environmental changes by fitness-induced attractor selection," PLoS ONE, vol. 1, no. 1, article e49, 2006.

[4] E. Stolovicki, T. Dror, N. Brenner, and E. Braun, "Synthetic gene recruitment reveals adaptive reprogramming of gene regulation in yeast," Genetics, vol. 173, no. 1, pp. 75-85, 2006.

[5] S. Stern, T. Dror, E. Stolovicki, N. Brenner, and E. Braun, "Genome-wide transcriptional plasticity underlies cellular adaptation to novel challenge," Molecular Systems Biology, vol. 3, article 106, 2007.

[6] M. Isalan, C. Lemerle, K. Michalodimitrakis et al., "Evolvability and hierarchy in rewired bacterial gene networks," Nature, vol. 452, no. 7189, pp. 840-845, 2008.

[7] S. Gama-Castro, V. Jiménez-Jacinto, M. Peralta-Gil et al., "RegulonDB (version 6.0): gene regulation model of Escherichia coli K-12 beyond transcription, active (experimental) annotated promoters and Textpresso navigation," Nucleic Acids Research, vol. 36, supplement 1, pp. D120-D124, 2008.

[8] T. M. Henkin and C. Yanofsky, "Regulation by transcription attenuation in bacteria: how RNA provides instructions for transcription termination/antitermination decisions," BioEssays, vol. 24, no. 8, pp. 700-707, 2002.

[9] F. Jacob and J. Monod, "Genetic regulatory mechanisms in the synthesis of proteins," Journal of molecular biology, vol. 3, pp. 318-356, 1961.
[10] H. H. McAdams, B. Srinivasan, and A. P. Arkin, "The evolution of genetic regulatory systems in bacteria," Nature Reviews Genetics, vol. 5, no. 3, pp. 169-178, 2004.

[11] T. Suzuki, A. Kashiwagi, I. Urabe, and T. Yomo, "Inherent characteristics of gene expression for buffering environmental changes without the corresponding transcriptional regulations," Biophysics, vol. 2, pp. 63-70, 2006.

[12] M. Acar, J. T. Mettetal, and A. van Oudenaarden, "Stochastic switching as a survival strategy in fluctuating environments," Nature Genetics, vol. 40, no. 4, pp. 471-475, 2008.

[13] E. Kussell and S. Leibler, "Phenotypic diversity, population growth, and information in fluctuating environments," Science, vol. 309, no. 5743, pp. 2075-2078, 2005.

[14] M. B. Elowitz, A. J. Levine, E. D. Siggia, and P. S. Swain, "Stochastic gene expression in a single cell," Science, vol. 297, no. 5584, pp. 1183-1186, 2002.

[15] E. L. Haseltine and F. H. Arnold, "Synthetic gene circuits: design with directed evolution," Annual Review of Biophysics and Biomolecular Structure, vol. 36, pp. 1-19, 2007.

[16] B. W. Ying, Y. Ito, Y. Shimizu, and T. Yomo, "Refined method for the genomic integration of complex synthetic circuits," Journal of Bioscience and Bioengineering, vol. 110, no. 5, pp. 529-536, 2010.

[17] B. S. Stevenson and T. M. Schmidt, "Life history implications of rRNA gene copy number in Escherichia coli," Applied and Environmental Microbiology, vol. 70, no. 11, pp. 6670-6677, 2004.

[18] Y. Mileyko, R. I. Joh, and J. S. Weitz, "Small-scale copy number variation and large-scale changes in gene expression," Proceedings of the National Academy of Sciences of the United States of America, vol. 105, no. 43, pp. 16659-16664, 2008.

[19] Y. Ito, H. Toyota, K. Kaneko, and T. Yomo, "How selection affects phenotypic fluctuation," Molecular Systems Biology, vol. 5, article 264, 2009.

[20] T. Baba, T. Ara, M. Hasegawa et al., "Construction of Escherichia coli K-12 in-frame, single-gene knockout mutants: the Keio collection," Molecular Systems Biology, vol. 2, article 2006.0008, 2006.

[21] K. A. Datsenko and B. L. Wanner, "One-step inactivation of chromosomal genes in Escherichia coli K-12 using PCR products," Proceedings of the National Academy of Sciences of the United States of America, vol. 97, no. 12, pp. 6640-6645, 2000.

[22] T. S. Gardner, C. R. Cantor, and J. J. Collins, "Construction of a genetic toggle switch in Escherichia coli," Nature, vol. 403, no. 6767, pp. 339-342, 2000.

[23] M. B. Elowitz and S. Leibier, "A synthetic oscillatory network of transcriptional regulators," Nature, vol. 403, no. 6767, pp. 335-338, 2000.

[24] S. Tsuru, J. Ichinose, A. Kashiwagi, B. W. Ying, K. Kaneko, and T. Yomo, "Noisy cell growth rate leads to fluctuating protein concentration in bacteria," Physical Biology, vol. 6, no. 3, Article ID 036015, 2009.

[25] S. Tsuru, N. Yasuda, Y. Murakami et al., "Adaptation by stochastic switching of a monostable genetic circuit in Escherichia coli," Molecular Systems Biology, vol. 7, p. 493, 2011.

[26] E. B. Keller and J. M. Calvo, "Alternative secondary structures of leader RNAs and the regulation of the trp, phe, his, thr, and leu operons," Proceedings of the National Academy of Sciences of the United States of America, vol. 76, no. 12, pp. 6186-6190, 1979. 
[27] L. L. Searles, S. R. Wessler, and J. M. Calvo, "Transcription attenuation is the major mechanism by which the leu operon of Salmonella typhimurium is controlled," Journal of Molecular Biology, vol. 163, no. 3, pp. 377-394, 1983.

[28] S. R. Wessler and J. M. Calvo, "Control of leu operon expression in Escherichia coli by a transcription attenuation mechanism," Journal of Molecular Biology, vol. 149, no. 4, pp. 579-597, 1981.

[29] E. M. Ozbudak, M. Thattal, H. N. Lim, B. I. Shraiman, and A. van Oudenaarden, "Multistability in the lactose utilization network of Escherichia coli," Nature, vol. 427, no. 6976, pp. 737-740, 2004.

[30] A. Kashiwagi, T. Sakurai, S. Tsuru, B. W. Ying, K. Mori, and T. Yomo, "Construction of Escherichia coli gene expression level perturbation collection," Metabolic Engineering, vol. 11, no. 1, pp. 56-63, 2009.

[31] R. Ihaka and R. Gentleman, "R: a language for data analysis and graphics," Journal of Computational and Graphical Statistics, vol. 5, no. 3, pp. 299-314, 1996. 

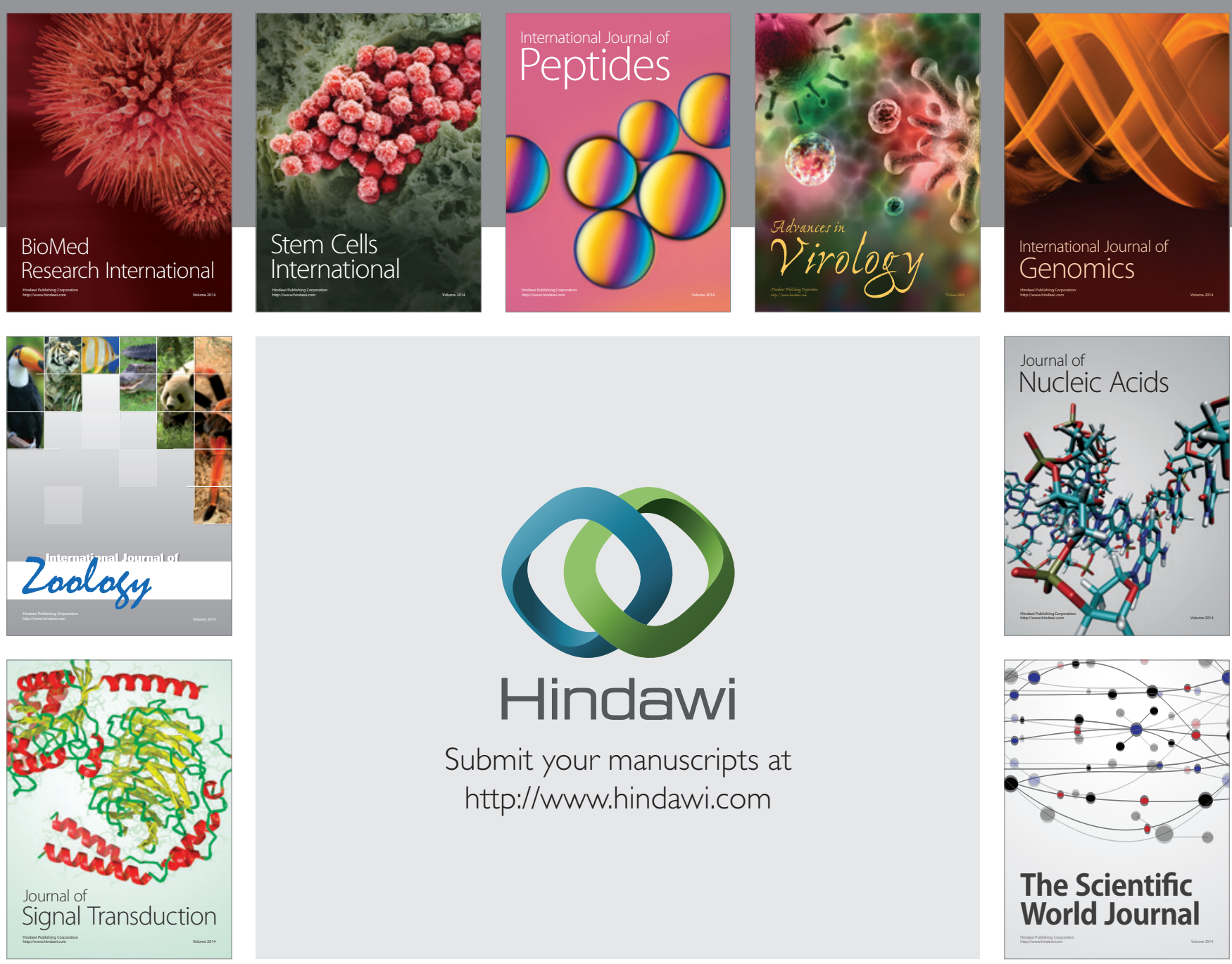

Submit your manuscripts at

http://www.hindawi.com
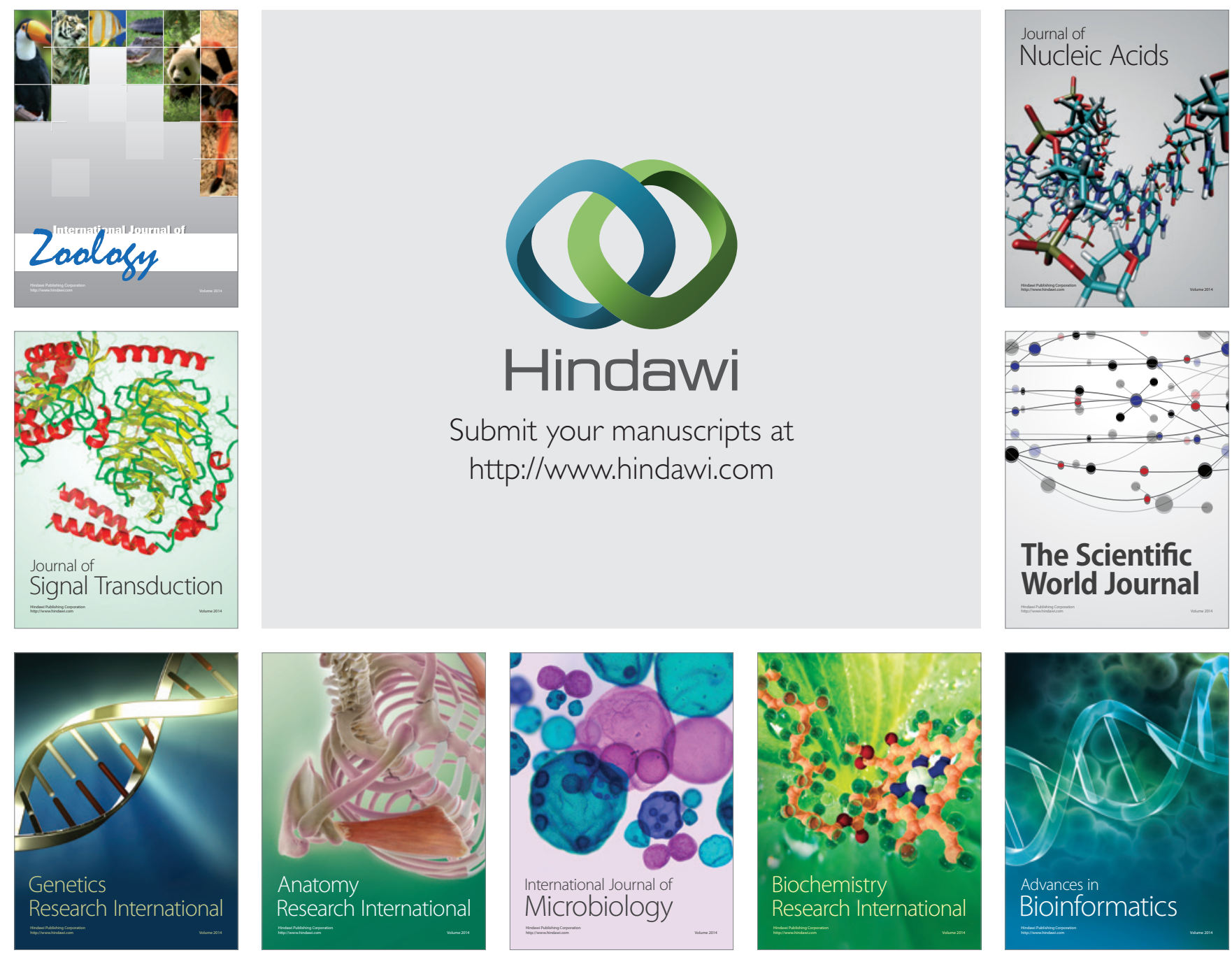

The Scientific World Journal
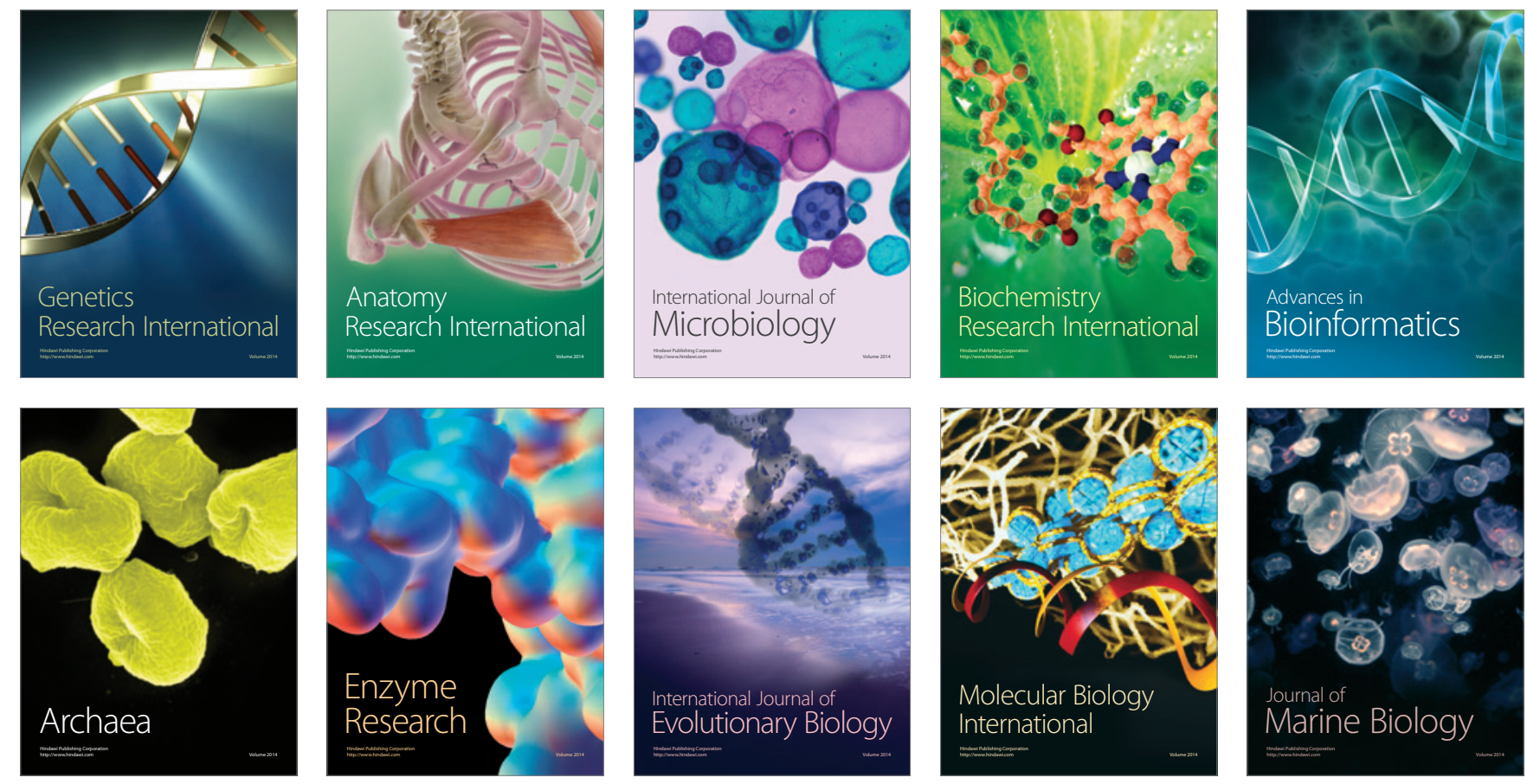